\title{
Solubilisation of Hydrophobic Drugs by Saponins
}

\author{
Z. VINAROV*, DENITSA RADEVA, V. KATEV, SLAVKA TCHOLAKOVAAND N. DENKOV \\ Department of Chemical and Pharmaceutical Engineering, Faculty of Chemistry and Pharmacy, Sofia University, 1164 Sofia, \\ Bulgaria
}

Vinarov et al.: Solubilisation of hydrophobic drugs by saponins

\begin{abstract}
Poor aqueous solubility limits the bioavailability of hydrophobic drugs. Thus, there is an increasing interest in new micelle-forming, drug-solubilizing molecules that can offer improved effectiveness and safety. The effect of 13 saponin extracts on the solubility of fenofibrate and danazol was studied and the relationship between saponin molecular structure and drug solubilisation capacity was assessed. Drug solubility was measured by high-performance liquid chromatography and saponin solubilisation capacity was compared to the conventional surfactant Brij-35. Saponins from Quillaja saponaria and Camellia oleifera improved the aqueous solubility of danazol and fenofibrate by more than two orders of magnitude. For danazol, the solubilisation capacity of the best saponins was 2-3 times higher than Brij-35, whereas for fenofibrate, it was slightly lower than the reference surfactant. Both drugs were solubilized very effectively by bidesmosidic oleanane saponins, whereas dammarane, mono- and tri-desmosidic oleanane saponins and steroid (furostanol and spirostanol) saponins had no effect on drug solubility. Exceptions were fenusterol, a furostanol saponin and escin, a monodesmosidic oleanane saponin, which solubilised danazol only.
\end{abstract}

Key words: Saponin, drug solubility, micelles, solubilisation

More than $40 \%$ of the orally administered immediaterelease drug products are characterized by poor aqueous solubility ${ }^{[1]}$. The slow and incomplete dissolution of such drugs in the gastrointestinal fluids limits their oral bioavailability and is one of the main problems in drug development ${ }^{[2,3]}$. One of the classical approaches to improve the aqueous solubility of hydrophobic drugs, which is still being widely used in the pharmaceutical industry, is to solubilize them in surfactant micelles ${ }^{[4,5]}$.

The surfactants most frequently used in conventional oral dosage forms are synthetic, such as polysorbates, sortbitan esters, polyoxyethylene alkyl ethers and sodium lauryl sulphate ${ }^{[6-8]}$. However, there is an increasing interest in finding new micelle-forming, drugsolubilizing molecules that offer improved drug-loading capacity, lower toxicity and greater drug stability ${ }^{[9-11]}$. One of the approaches that have attracted significant attention is the polymeric micelles ${ }^{[12]}$. The latter are formed by block-copolymers with great structural and functional diversity, which allows the development of non-toxic, targeted, stimuli-responsive drug delivery vehicles for a broad variety of drugs ${ }^{[12-14]}$. Another recent finding is that the diglutamic acid-fatty acid family of surfactants enhanced strongly the solubility of poorly water-soluble anticancer drugs, while

*Address for correspondence

E-mail: zv@dce.uni-sofia.bg

July-August 2018

Indian Journal of Pharmaceutical Sciences maintaining low toxicity profile ${ }^{[11]}$. Promising results were obtained also with hydrophobically-modified polysaccharides: amphiphilic molecules formed by conjugation of a polysaccharide (e.g. pullulan, cellulose, dextran, heparin) with a hydrophobic moiety (e.g. fatty acids, bile acids, cholesterol, polylactides) ${ }^{[15]}$. For example, amphiphilic carboxymethylpullulans, $\mathrm{CMP}_{49} \mathrm{C}_{8}$ and $\mathrm{CMP}_{12} \mathrm{C}_{8}$ were shown to solubilize docetaxel as effectively as the Tween 80:ethanol:water vehicle used in a commercial formulation (Taxotere ${ }^{\circledR}$, Aventis Pharmaceuticals Inc.), and also provided lower cytotoxicity ${ }^{[10]}$.

In the current article, the solubilisation of hydrophobic drugs by saponins was investigated. Saponins are a diverse family of natural surfactants, which are present in a wide variety of plants ${ }^{[16,17]}$. The saponin molecules consist of a hydrophobic region, called aglycone, which is linked to one or several oligosaccharide (sugar) chains that build the hydrophilic part of the molecule.

This is an open access article distributed under the terms of the Creative Commons Attribution-NonCommercial-ShareAlike 3.0 License, which allows others to remix, tweak, and build upon the work non-commercially, as long as the author is credited and the new creations are licensed under the identical terms

Accepted 25 June 2018

Revised 30 November 2017

Received 14 April 2017

Indian J Pharm Sci 2018;80(4):709-718 
The saponins are classified depending on the type of aglycone (triterpenoid or steroid) and the number of attached sugar chains. Most of the saponins have two sugar chains (bidesmosidic saponins), some have one sugar chain (monodesmosidic type) and in rare cases, three sugar chains.

Because of their amphiphilic nature, saponin molecules form micelles in aqueous solutions. The size, shape, and structure of the saponin micelles depend on their plant origin, temperature, $\mathrm{pH}$, electrolyte concentration, and so forth ${ }^{[18-21]}$. Experiments showed that the micellar solutions of saponins, extracted from the tree Quillaja saponaria, are able to efficiently solubilize large hydrophobic molecules such as cholesterol, phytosterols, phenanthrene, and others ${ }^{[20-22]}$. A number of studies have demonstrated the ability of saponins to solubilize molecules with similar structure like other saponins or aglycones ${ }^{[23-27]}$. Walthelm et al. found that saponins did not increase strongly or consistently the solubility of several hydrophilic $(\log \mathrm{P}<2)$ model compounds from plant origin ${ }^{[28]}$. However, so far there is no systematic study on the solubilisation of hydrophobic drugs by saponins.

Saponins are also known to act as intestinal permeation enhancers $^{[29]}$. Model experiments with Caco-2 cells showed increased permeability in presence of glycyrrhizin ${ }^{[30]}$ or Quillaja saponins ${ }^{[31]}$, via a transcellular mechanism. Again, water-soluble model compounds were used in these studies and thus the effect of saponins on the aqueous solubility and its influence on the permeation was not assessed.

When screening for a novel pharmaceutical excipient, one of the major concerns is toxicity. Significant advantage of saponins is that they are very poorly absorbed upon oral ingestion ${ }^{[32]}$, thus reducing the probability for systemic toxic effects. For example, saponin extracts from $Q$. saponaria are approved as a food additive in the USA (FEMA no. 2973) and EU (E999), and find application as emulsifiers and foamers in food technology.

The maximum level of use of $Q$. saponaria extracts in foods is relatively high, $500 \mathrm{mg} / \mathrm{kg}$, which further confirms their possible application in drug delivery. Ryokucha saponins from Camellia sinensis (green tea) demonstrated lower oral toxicity, compared to Quillaja saponins in rats ${ }^{[33]}$. Other saponins obtained from Dioscorea zingiberensis, Amaranthus cruentus and Medicago sativa also showed low toxicity upon oral ingestion in rats ${ }^{[34-36]}$.
In veterinary medicine, saponins from $Q$. saponaria are used as potent adjuvants for vaccines ${ }^{[37]}$. This breakthrough was allowed by the thorough investigation of the haemolytic properties of Quillaja saponins, which demonstrated that haemolysis depends on the type of saponin fraction ${ }^{[38]}$. Thus, saponins could be used also for other routes of drug delivery, different from oral, after sufficient purification and fractionation.

Therefore, the major aims of this study were to characterize the ability of saponin extracts to enhance drug solubility and to investigate the relationship between saponin molecular structure and drug solubilisation capacity. The model poorly-water soluble drugs we chose to study were fenofibrate and danazol, which are class-II compounds, according to the Biopharmaceutical classification system (BCS), and thus have very low aqueous solubility and good membrane permeability ${ }^{[1]}$. Furthermore, fenofibrate and danazol are very frequently used as model poorly-water soluble drugs in research, which can allow comparison between the saponins and other strategies for enhancement of oral absorption ${ }^{[39-41]}$. The effectiveness of the saponin extracts was compared to a standard surfactant- polyoxyethylene (23) lauryl ether (Brij-35), which has low toxicity and is used in a variety of drug delivery applications ${ }^{[22-44]}$. The strength of the saponin-drug interactions was assessed by calculating the change of the standard chemical potential of the drug upon its solubilisation in saponin micelles.

\section{MATERIALS AND METHODS}

A total of 13 saponin extracts from 10 different plants were tested as solubilizing agents. We chose to study a wide range of saponins, which differ in the type of the aglycone and/or the number and type of oligosaccharide chains, in order to investigate the relationship between saponin molecular structure and drug solubilisation capacity. Table $1^{[45-57]}$ contains information about all studied saponin extracts, trade name, abbreviation used in the text, supplier, origin of the plant extract, part of the plant which was processed, concentration of saponins in each extract, predominating chemical structure and critical micelle concentration (CMC). Five of the studied extracts were highly purified with saponin content above $80 \%$ and 8 were crude extracts (saponin content between 20 and $53 \%$ ). The crude extracts were composed by a complex mixture of saponins and other natural components as polyphenols and, carbohydrates ${ }^{[58]}$. The role of the crude extracts in the study was to provide 
www.ijpsonline.com

TABLE 1: MAIN PROPERTIES OF THE STUDIED SAPONIN EXTRACTS

\begin{tabular}{|c|c|c|c|c|c|c|c|}
\hline Trade name & $\begin{array}{l}\text { Abbreviation } \\
\text { used in text }\end{array}$ & Plant species & $\begin{array}{l}\text { Extracted } \\
\text { from }\end{array}$ & Supplier & $\begin{array}{c}\text { Saponin } \\
\text { content, } \\
\text { wt } \%\end{array}$ & $\begin{array}{c}\text { General } \\
\text { chemical } \\
\text { structure }\end{array}$ & $\begin{array}{l}\text { CMC, } \\
\text { wt \% }\end{array}$ \\
\hline \multicolumn{8}{|c|}{ Purified saponins } \\
\hline Fraction C & $\mathrm{FC}$ & Quillaja saponaria & Bark & Desert King & 99 & $\mathrm{~B}^{[46]}$ & $0.004^{c}$ \\
\hline Tea saponin & TS & Camelia oleifera & Seeds & $\begin{array}{l}\text { Zhejiang } \\
\text { Yuhong }\end{array}$ & 96.2 & $(A+B)^{[47]}$ & $0.015^{\mathrm{a}}$ \\
\hline $\begin{array}{l}\text { Glycyrrhizic acid } \\
\text { ammonium salt }\end{array}$ & LC & Glycyrrhiza glabra & Roots & Sigma & $\geq 95$ & - & - \\
\hline Escin & ESC & $\begin{array}{c}\text { Aesculus } \\
\text { hippocastanum }\end{array}$ & Seeds & Sigma & $\geq 95$ & $\mathrm{~A}^{[48]}$ & $0.008^{b}$ \\
\hline Ginsenosides & GS & Panax ginseng & Roots & $\begin{array}{l}\text { Xianyang Hua } \\
\text { Yue B.E. }\end{array}$ & 80 & $D^{[49,50]}$ & $0.019^{b}$ \\
\hline \multicolumn{8}{|c|}{ Crude saponin extracts } \\
\hline $\begin{array}{l}\text { Berry saponin } \\
\text { concentrate }\end{array}$ & BSC & Sapindus mukorossi & Fruits & Eco. surf. & 53 & $(A+B)^{[51]}$ & $0.1^{b}$ \\
\hline Fenusterols & FEN & $\begin{array}{l}\text { Trigonella foenum- } \\
\text { graecum }\end{array}$ & Seeds & Sabinsa corp. & 50 & $\mathrm{~F}^{[52,53]}$ & $0.089^{b}$ \\
\hline Sapindin & SAP & Sapindus trifoliatus & Fruits & Sabinsa corp. & 50 & $(A+B)^{[51,54]}$ & $0.03^{b}$ \\
\hline $\begin{array}{l}\text { Tribulus terrestris } \\
\text { extract }\end{array}$ & TT & Tribulus terrestris & Herb & Sabinsa corp. & 45 & $(E+F)^{[55,56]}$ & $0.048^{b}$ \\
\hline Prototrib & PT & Tribulus terrestris & Herb & Sabinsa corp. & 40 & $(E+F)^{[55,56]}$ & - \\
\hline $\begin{array}{l}\text { Ayurvedic saponin } \\
\text { concentrate }\end{array}$ & ASC & Acacia concinna & Pods & Eco. surf. & 30 & $C^{[57]}$ & $0.3^{\mathrm{b}}$ \\
\hline Quillaja dry 100 NP & QD & Quillaja saponaria & Bark & Desert King & 26 & $\mathrm{~B}^{[46]}$ & $0.004^{a}$ \\
\hline Horse chestnut extract & $\mathrm{HC}$ & $\begin{array}{c}\text { Aesculus } \\
\text { hippocastanum }\end{array}$ & Seeds & $\begin{array}{l}\text { Xi'an Biof } \\
\text { Bio-tech. }\end{array}$ & 20 & $\mathrm{~A}^{[48]}$ & $0.146^{\mathrm{b}}$ \\
\hline
\end{tabular}

'a' Determined by light-scattering in this study; 'b' determined by surface tension by Golemanov et al. ${ }^{[45]}$; 'b' assumed to be the same as for QD

information on the solubilisation capacity of saponin types that were not available as highly purified extracts, and also to compare the solubilisation properties of pairs of crude and purified extracts, obtained from the same plant. The latter was necessary in order to check if saponin molecules were the ones that govern drug solubilisation. Quillaja dry 100 NP (QD) and fraction C (FC) extracts were obtained from $Q$. saponaria and contained 26 and $99 \%$ Quillaja saponins, respectively, whereas horse chestnut extract (HC) and escin (ESC) are Aesculus hippocastanum extracts containing 20 and $95 \%$ saponins, respectively. The saponin content of each extract in Table 1 was taken into account when calculating the saponin concentration in the solubilisation experiments.

A classical surfactant of the alcohol ethoxylate typepolyoxyethylene (23) lauryl ether, Brij-35 (SigmaAldrich) was used as a reference for comparison of the effectiveness of saponin solutions. Two hydrophobic drugs were used, both products of Sigma-Aldrich with purity $\geq 98 \%$, fenofibrate (molecular weight, $\mathrm{MW}=360.8 \mathrm{~g} / \mathrm{mol}$ ) and danazol (MW=337.5 $\mathrm{g} / \mathrm{mol})$. Both drugs have very low aqueous solubility i.e.
1 and $0.8 \mu \mathrm{g} / \mathrm{ml}$ for danazol ${ }^{[7]}$ and fenofibrate ${ }^{[8]}$, respectively.

Mobile phase solvents for high-performance liquid chromatography (HPLC) analysis included methanol (HPLC grade, $99.9 \%$, Sigma-Aldrich) and deionized water, filtered through $450 \mathrm{~nm}$ nylon filter. All aqueous solutions and phases were prepared using deionized water from water-purification system Elix 3 (Millipore, USA).

\section{Drug solubility in micellar solutions:}

To determine the equilibrium drug solubility in presence of saponins, excess amount of drug $(1.5 \mathrm{mg} / \mathrm{ml})$ was added to a freshly prepared solution $(10 \mathrm{ml})$ of saponin extract or Brij-35. The concentration of saponin or Brij-35 in most of the experiments was $0.5 \mathrm{wt} \%$. The mixture was then stirred with a magnetic stir bar at $400 \mathrm{rpm}$ for $24 \mathrm{~h}$ in a water bath, equipped with external temperature probe with an accuracy of $\pm 0.2^{\circ}$, set at $37.0^{\circ}$. After incubation, the suspension was filtered through $200 \mathrm{~nm}$ nylon syringe filters to eliminate all undissolved particles. These filters were pre-heated at $37^{\circ}$ in an incubator. The clear aqueous phases obtained after filtration were stored in HPLC 
vials that were thermostated at $37^{\circ}$ in a water bath. All samples were kept in the water bath at $37^{\circ}$ until they were analysed on the HPLC. Every step of the procedure before the HPLC analysis was performed at a constant temperature of $37 \pm 0.2^{\circ}$.

To check if equilibrium solubility is reached for $24 \mathrm{~h}$, the solubility of fenofibrate in $0.5 \%$ solutions of several saponins (QD; tea saponin, TS) was measured at 24, 48 and $72 \mathrm{~h}$. No significant difference in the results was observed, as a function of time. No chemical changes in the structure of the drugs occurred when they were solubilized in the saponin extract solutions: a single peak, identical with the drug standard was found in the HPLC chromatograms of all studied saponin extracts.

\section{Solubilisation capacity:}

The solubilisation capacity of the studied saponin extracts was defined as Eqn. $1^{[59]}, \chi=\left(\mathrm{S}_{\text {tot }}-\mathrm{S}_{\mathrm{w}}\right) /\left(\mathrm{C}_{\mathrm{s}}-\right.$ $\mathrm{CMC}) \times 1000$, where Stot is the measured molar drug solubility in the presence of saponin, $\mathrm{S}_{\mathrm{w}}$ is the intrinsic aqueous solubility of the drug, $\mathrm{C}_{\mathrm{s}}$ is the saponin molar concentration, and $\mathrm{CMC}$ is the critical micelle concentration of the saponin extract. This definition of the solubilisation capacity gives the number of drug molecules solubilized per 1000 saponin molecules. Note that the subtraction of $\mathrm{S}_{\mathrm{w}}$ and $\mathrm{CMC}$ from Stot and $\mathrm{C}_{\mathrm{s}}$, respectively, allows us to consider only the drug and saponin molecules that are incorporated in the micelles. To calculate the molar concentration of saponins the molecular mass of the predominating saponin in the extract was used. Although this approach is not completely accurate as the used extracts contain a mixture of saponins, it allows quantitative comparison of the molar solubilisation effectiveness of the different saponin extracts. The used average molecular mass of the saponins is as follows: $1131 \mathrm{~g} / \mathrm{mol}$ for ESC and $\mathrm{HC}, 1287 \mathrm{~g} / \mathrm{mol}$ for TS, $1650 \mathrm{~g} / \mathrm{mol}$ for $\mathrm{FC}$ and QD, $817 \mathrm{~g} / \mathrm{mol}$ for berry saponin concentrate (BSC), $55 \mathrm{~g} / \mathrm{mol}$ for sapindin (SAP) and $577 \mathrm{~g} / \mathrm{mol}$ for fenusterols (FEN).

As follows from Eqn. 1, the solubilisation capacity can be determined from experiments at constant saponin or surfactant concentration, as long as the dependence of $S_{\text {tot }}$ on $C_{\mathrm{S}}$ is linear ${ }^{[59]}$. Thus, most of the experiments for determination of solubilisation capacity were performed at constant saponin concentration of $0.5 \mathrm{wt} \%$.

\section{RESULTS AND DISCUSSION}

The solubilisation capacity of the purified and crude saponin extracts is presented in fig. 1A and
$\mathrm{B}$, respectively. In general, danazol was solubilized much more efficiently than fenofibrate by almost all of the studied saponin extracts, regardless of their saponin content. For both drugs, best solubilisation was observed in presence of $Q$. saponaria $(\mathrm{FC})$ and $C$. oleifera saponins (TS), whereas saponins from Glycyrrhiza glabra (glycyrrhizic acid ammonium salt, LC) and Panax ginseng (ginsenosides, GS) did not increase drug solubility to detectable levels (fig. 1A). Interestingly, the A. hippocastanum saponin ESC had very different solubilisation capacity depending on the type of drug molecule: fenofibrate was not solubilized at all, whereas there was strong effect for danazol, $\chi=23 \mathrm{mM} / \mathrm{M}$.

Similarly to the results obtained with purified saponins, best solubilisation of both studied drugs was obtained by $Q$. saponaria crude extract (QD, fig. 1B). The crude $A$. hippocastanum extract also displayed
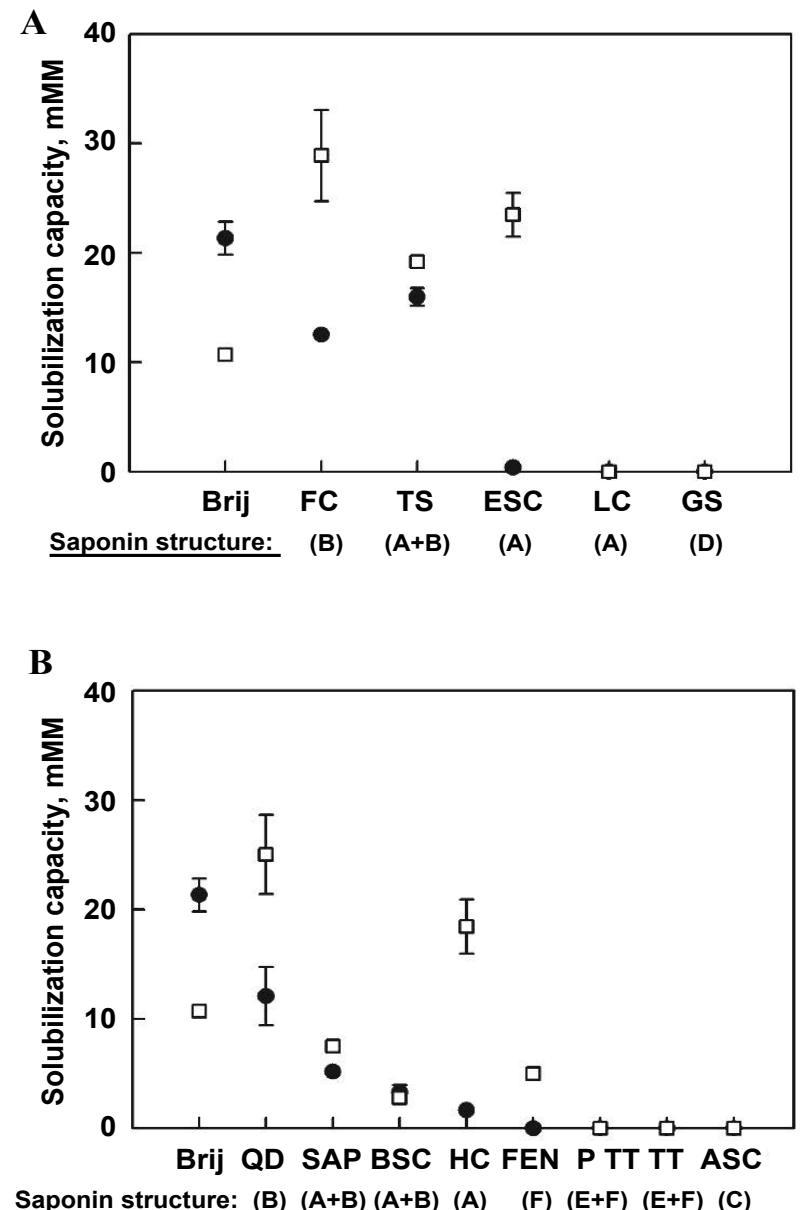

Saponin structure: (B) $(A+B)(A+B)(A) \quad(F)(E+F)(E+F)$ (C)

Fig. 1: Molar solubilisation capacity, $\chi$, of (A) purified saponins and $(B)$ crude saponin extracts for danazol and fenofibrate The results for the conventional surfactant Brij-35 are presented for the purpose of comparison. The results are averaged over at least two independent experiments. The error bars can be smaller than the symbols. $\square$ Danazol, $\bullet$ fenofibrate 
selectivity toward the type of drug molecule: very low solubilisation for fenofibrate, whereas danazol solubility was improved significantly. The latter finding is in accordance with the results obtained with pure ESC, fig. 1A. Some selectivity towards danazol was found also for Trigonella foenum-graecum saponin extract (FEN), although the measured danazol solubilisation capacity was small. Saponin extracts from Sapindus trifoliatus (SAP) and S. mukorossi (BSC) also had small effects on the solubility of both studied drugs, while no solubilisation at all was observed in presence of Acacia concinna (Ayurvedic saponin concentrate, ASC) and T. terrestris saponin extracts (Prototrib, PT and $T$. terrestris extract, TT).

The comparison between the danazol solubilisation efficiency of the saponins and the conventional surfactant shows that $\mathrm{FC}(\chi=29 \mathrm{mM} / \mathrm{M})$ and TS $(\chi=$ $19 \mathrm{mM} / \mathrm{M}$ ) have about 3-fold and 2-fold higher danazol solubilisation capacity than Brij-35 ( $\chi=11 \mathrm{mM} / \mathrm{M})$, respectively (fig. 1A). For fenofibrate, the solubilisation capacity of TS ( $\chi=16 \mathrm{mM} / \mathrm{M})$ was slightly lower than Brij-35 $(\chi=21 \mathrm{mM} / \mathrm{M})$. FC also displayed smaller fenofibrate solubilisation capacity $(\chi=13 \mathrm{mM} / \mathrm{M})$ than Brij-35.

As both of the drugs studied were high dose drugs (100-200 mg per single dose), they required significant amount of saponins for solubilisation. For example, $100 \mathrm{mg}$ dose of danazol can be formulated in a $7 \mathrm{wt}$ $\%$ Quillaja saponins solution with volume of $200 \mathrm{ml}$.
However, drugs with similar structure and properties, but much lower single dose, such as many of the female hormones used in oral contraception products, could be efficiently solubilized in saponin solutions. Such drugs, for example, are ethynylestradiol (0.03 $\mathrm{mg}$ single dose) or dienogest ( $2 \mathrm{mg}$ single dose). Thus, the studied saponins should be able to adequately solubilize low-dose drugs.

Several of the studied saponins increased significantly the solubility of both studied drugs (fig. 1). The best performing crude saponin extract QD managed to increase the aqueous solubility of danazol and fenofibrate by 150 and 100 times, respectively, at a concentration of $12 \mathrm{mM}$. These results are contrary to the findings of Walthelm et al., who showed that saponins with various structures and plant source have small effect on the aqueous solubility of a number of model compounds ${ }^{[28]}$. The major difference that can account for the discrepancy between the two studies is that the drugs used in our study are very hydrophobic $(\log \mathrm{P}>4)$, whereas the model compounds used by Walthelm et al. are relatively hydrophilic (log $\mathrm{P}<2$ ). Most likely, the hydrophobic nature of fenofibrate and danazol renders their incorporation in the saponin micelles energetically favourable, resulting in the measured strong increase of their aqueous solubility. Furthermore, the authors did not study Quillaja or tea saponins, while we showed that these are the most potent solubilizers.
(A)

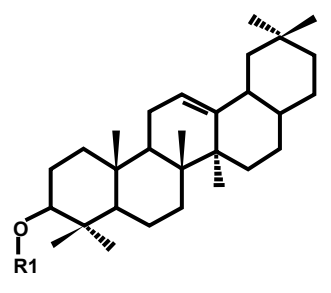

(B)

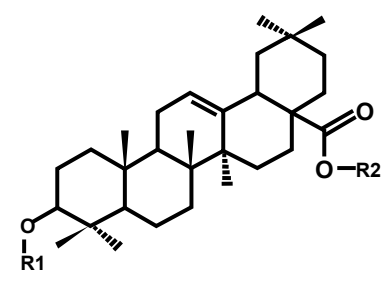

(C)

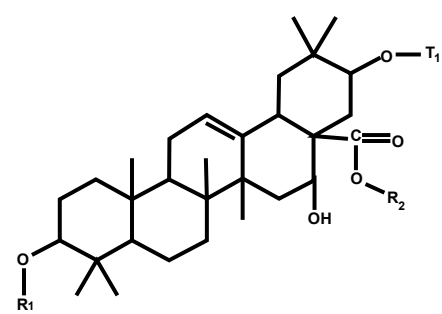

(D)

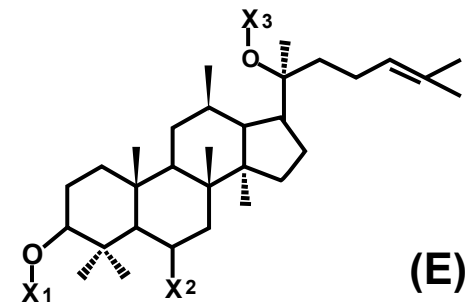

(E)

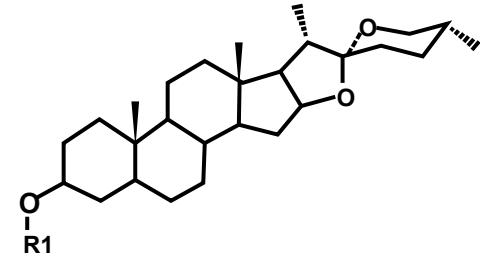

(F)

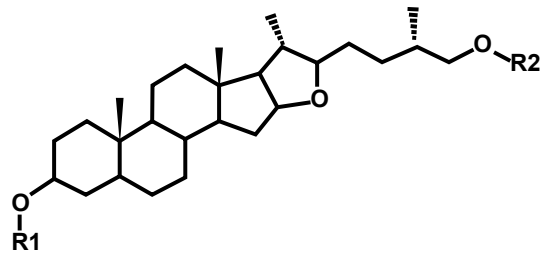

Fig. 2: Predominating saponin structures in the studied extracts, as referenced in Table 1

$R 1-R 2$, and X1-X3, designate sugar chains with different length and/or composition; X1-X3 can also designate $H$ atom; $T 1$ is menthiafolic acid quinovoside and/or xyloside. Monodesmosidic (A), bidesmosidic (B) and tridesmosidic (C) triterpenoids with oleanane type of aglycone. Mono- or bidesmosidic triterpenoids with dammarane type of aglycone (D). Steroid saponins of the spirostanol (E) and furastanol (F) type 
The molecular-level interpretation of the obtained results was not a trivial task for a number of reasons. One of the main issues was to check whether the saponins are responsible for micellization and, consequently, for drug solubilisation. This was due to the fact that part of the studied saponin extracts are crude (saponin content of 20 to $53 \%$ ) and contain a variety of other substances. Furthermore, each extract contains a wide variety of saponin molecules, which differ mainly by the number and type of sugar chains, but also by the number of double bonds and methyl groups in the aglycone.

Thus, the following discussion will be focused on two questions: 1. do saponins govern the solubilisation properties of the solutions? 2. How do the molecular structures of the drugs and the saponins (type of aglycone, number of sugar chains) determine the solubilisation capacity? Finally, the thermodynamic aspects of drug solubilisation by saponins, emphasizing on the energy gain of the system upon incorporation of a drug molecule in the micelle, will be addressed.

All of the studied saponin extracts were standardized with respect to their saponin content by the manufacturer. However, they usually contain additional substances: for example, $Q$. saponaria extracts contain polyphenols, phenolic acids, polysaccharides and calcium $^{[58]}$. It was therefore necessary to validate that the measured enhancement of drug solubility was due to drug solubilisation in saponin micelles.

To address this issue, the solubilisation capacity of pairs of crude (20-26\% saponins) and purified (95$99 \%$ saponins) extracts obtained from two different plants (Q. saponaria and A. hippocastanum) was compared. Another important difference between the two pairs of saponin extracts was that they were produced by extraction of very different parts of the plants: seeds for A. hippocastanum and bark for $Q$. saponaria. Thus, the crude extracts should have very different phytochemical profile of concomitant substances (e.g. lipids and polysaccharides for the seeds; tannins and catechins for the bark).

The obtained results clearly showed that crude and purified saponin extracts have the same solubilisation capacity. Therefore, it could reasonably be concluded that the ability of saponin extracts to enhance drug solubility was due exclusively to the presence of saponin molecules. The other substances in the crude extracts appeared to have insignificant effect on the measured drug solubilisation.

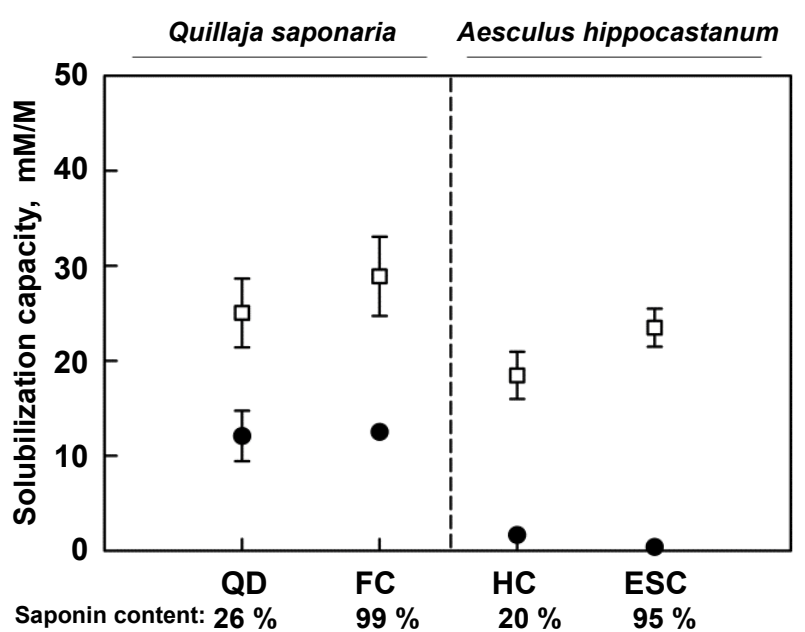

Fig. 3: Comparison of the molar solubilization capacity, $\chi$, of purified and crude saponin extracts of Quillaja saponaria and Aesculus hippocastanum for danazol and fenofibrate

The results are averaged over at least two independent experiments. The error bars can be smaller than the symbols. $\square$ Danazol, • fenofibrate

The studied saponins have triterpenoid or steroid structures. The triterpenoid saponins have oleanane (structures A-C) or dammarane (structure D) type of aglycones, whereas the steroid saponins are of spirostanol (structure E) or furostanol (structure F) type. The number of sugar chains, attached to the aglycone, varies between 1 and 3 for the different saponins (figs. 2 and 3, Table 1).

The experiments performed with highly purified extracts (fig. 1A) clearly demonstrated that bidesmosidic oleanane saponins (structure B) efficiently solubilised both drugs studied. In contrast, dammarane and monodesmosidic oleanane saponins (structures A and D) had no effect on drug solubility. Exception is ESC, which showed high solubilisation capacity for danazol only.

The results obtained with crude saponin extracts (fig. 1B) provided additional information about the structure-activity relationship of the saponins. The general picture is in excellent agreement with the trends observed for the highly purified extracts: saponins with structure B (bidesmosidic oleanane) are most efficient in drug solubilisation, whereas all other studied saponins (mono- and tri-desmosidic oleanane, dammarane and steroid saponins) have no effect on drug solubility. It was found that higher drug solubilisation capacity is related to higher solvent accessible surface area of the best saponins.

The general observation that bidesmosidic oleanane saponins have the highest solubilisation efficiency is 
strictly valid for fenofibrate, whereas for danazol there are two exceptions. The first one is the solubilisation of danazol by HC and ESC, which are both extracts of A. hippocastanum and contain monodesmosidic oleanane saponins as the primary component. The other is the effect of FEN (a furostanol saponin), which could be explained by the high structural compatibility between the steroidal structure of this saponin and danazol, which is also steroidal in nature. ${ }^{1} \mathrm{H}$-nuclear magnetic resonance spectroscopy of danazol and fenofibrate solubilized in FEN or ESC micelles would provide useful structural information about the location and orientation of the drug molecule in the micelles and could reveal the reason for the selectivity of these saponins towards danazol. However, such investigation is beyond the scope of the present study.

In summary, both the drugs studied were solubilized very effectively by triterpenoid saponins of the oleanane type with two sugar chains (bidesmosidic oleanane saponins). No solubilisation was observed for dammarane, mono- and tri-desmosidic oleanane saponins and steroid (furostanol and spirostanol) saponins. Exception was danazol, which was solubilized preferably by a furostanol saponin extract (FEN) and one monodesmosidic oleanane saponin (ESC). In respect to the effect of drug structure on solubilisation, it was shown that danazol is solubilized much more efficiently than fenofibrate by almost all of the studied saponin extracts (fig. 1). The latter could be due to the structural similarity between the steroidal danazol molecule and the triterpenoid/steroid saponin aglycones. In contrast to these non-aromatic structures, fenofibrate contains a large planar moiety consisting of two aromatic rings with a carbonyl group in the middle ( $\mathrm{Ph}-\mathrm{CO}-\mathrm{Ph}$ ), which is apparently more difficult to pack in between the saponin molecules in the micelles.
The obtained results for the transfer energies of drug molecules from crystal to solution, $\Delta \mu^{0}{ }_{c r / s o l}$ and from aqueous environment to micelles, $\Delta \mu_{w / m i c}^{0}$, are presented in Table 2. These transfer energies represent the change in the standard chemical potential of the drug molecule upon its transfer from one media (drug crystal or aqueous molecular solution) into the surfactant micelles. For both studied drugs, the transfer of one drug molecule from the aqueous environment to the micelle was associated with a considerable energy gain, which was similar for all studied saponins: $\Delta \mu_{w / m i c}^{0}=$ -11 to $-13 \times k T$, where $k T$ is the thermal energy. These similar values of the energy gain for solubilisation of two chemically very different molecules (danazol and fenofibrate) suggest that the drug-saponin interactions in the micelle are of similar type and magnitude. In support of this explanation, the molecular weight of the studied drugs is comparable ( 360.8 and $337.5 \mathrm{~g} / \mathrm{mol}$ for fenofibrate and danazol, respectively) and it is known that the van der Waals and hydrophobic interactions are both approximately proportional to the molecular size.

For danazol, the decrease in $\Delta \mu_{w / m i c}^{0}$ for the best saponin extracts FC, TS and QD (13.2, 12.8 and $13.0 \times k T$, respectively) was higher than that of the synthetic surfactant Brij-35 (12.2×kT), demonstrating once again the better effectiveness of these saponins, compared to the reference surfactant. The energy gain upon fenofibrate solubilisation in the micelle of Brij-35 $(13.2 \times k T)$ is greater than that of FC and TS (12.6 and $12.9 \times k T$, respectively), which is in agreement with the better solubilisation of fenofibrate by the reference surfactant.

The transfer energy of the drug molecule from the drug crystal into the micellar solution, $\Delta \mu_{c r s o l}^{0}$, was positive, which signifies an increase in the energy

TABLE 2: TRANSFER ENERGIES FOR FENOFIBRATE AND DANAZOL

\begin{tabular}{|c|c|c|c|c|}
\hline \multirow{3}{*}{ Saponin extract } & \multicolumn{4}{|c|}{ Transfer energy of drug molecule } \\
\hline & \multicolumn{2}{|c|}{ From crystal to micelle, $\Delta \mu_{c r / s o l}^{0} / k T$} & \multicolumn{2}{|c|}{ From water to micelle, $\Delta \mu_{c r / s o l}^{o} / k T$} \\
\hline & Fenofibrate & Danazol & Fenofibrate & Danazol \\
\hline BSC & 5.7 & 5.9 & -11.3 & -10.9 \\
\hline ESC & 7.9 & 3.5 & -9.2 & -13.3 \\
\hline FC & 4.4 & 3.6 & -12.6 & -13.2 \\
\hline FEN & n.a. & 5.3 & n.a. & -11.4 \\
\hline $\mathrm{HC}$ & 6.6 & 4.0 & -10.5 & -12.7 \\
\hline QD & 4.2 & 3.7 & -12.8 & -13.0 \\
\hline SAP & 5.3 & 4.9 & -11.8 & -11.9 \\
\hline TS & 4.1 & 4.0 & -12.9 & -12.8 \\
\hline Brij-35 & 3.9 & 4.5 & -13.2 & 12.2 \\
\hline
\end{tabular}

The transfer energy of drug molecule from crystal to pure water, $\Delta \mu_{\mathrm{cr} / \mathrm{sol}}^{0}$, is $17.04 \mathrm{kT}$ and $16.75 \mathrm{k} T$ for fenofibrate and danazol, respectively, where $k T \approx 4.1 \times 10^{-21} \mathrm{~J}$ is the thermal energy 
of the molecule upon this transfer. For both studied drugs, the energy increase upon transfer of a molecule from crystal into molecular aqueous solution (without micelles) was very strong, $\approx 17 \times k T$. This effect is due to the unfavourable interactions between the hydrophobic drug molecules and the polar water molecules, and causes the very low aqueous solubility, 1 and $0.8 \mu \mathrm{g} / \mathrm{ml}$ for danazol ${ }^{[7]}$ and fenofibrate ${ }^{[8]}$, respectively. In contrast, the energy increase was three to five times lower when the drug molecule was transferred from the solid phase into the micellar solution of saponins or Brij-35, $\Delta \mu_{c r s o l}^{0}=3$ to $6 \times k T$, for both studied drugs. The presence of hydrophilic and hydrophobic regions in the micelles allows the hydrophobic drug molecule to minimize its energy in respect to the hydrophobic, van der Waals and steric interactions (packing). As a consequence, the increase of $\Delta \mu^{0}{ }_{c r / s o l}$ was much smaller when the drug molecule was transferred from crystal to micelle environment, compared to the crystal-water transfer.

The drug molecule can be solubilized in different regions of the micelle ${ }^{[59,60]}$ such as in its hydrophobic core, in the palisade layer, on the outer micellar surface or within the polyoxyethylene chains (for Brij-35). Determination of the exact locus of drug solubilisation required extensive knowledge of the molecular organization of saponin micelles, which is beyond the scope of the present study.

To conclude, the effect of 13 saponin extracts obtained from 10 different plants on the solubilisation of two poorly-water soluble drugs, fenofibrate and danazol was systematically studied. Two highly purified saponin extracts (FC and TS) improved the solubility of both danazol and fenofibrate considerably. The latter result is encouraging, as FC was already studied in clinical trials as a vaccine adjuvant in humans ${ }^{[61]}$ and is known to have low oral toxicity. It is already in use as a food additive in USA, (FEMA no. 2973) and (EU E999). In addition, ESC saponin was found to enhance greatly danazol solubility, while having no effect for fenofibrate.

Both of the drugs studied were solubilized very effectively by bidesmosidic triterpenoid saponins of the oleanane type. Steroid saponins showed no effect on fenofibrate solubility, whereas danazol was solubilized by a steroid saponin of the furostanol type. Calculation of the transfer energy of drug molecule from aqueous environment to micelle, $\Delta \mu^{0}{ }_{w / m i c}$, showed that drug solubilisation in saponin micelles is driven by a considerable energy gain, $\Delta \mu_{w / m i c}^{0}=-11$ to $-14 \times k T$.

\section{Acknowledgements:}

The authors gratefully acknowledge student Nevse Darakchi, who performed few of the solubility measurement experiments.

\section{Conflicts of interest:}

There are no conflicts of interest.

\section{REFERENCES}

1. Takagi T, Ramachandran C, Bermejo M, Yamashita S, Yu LX, Amidon GL. A provisional biopharmaceutical classification of the top 200 oral drug products in the United States, Great Britain, Spain, and Japan. Mol Pharm 2006;3(6):631-43.

2. Lipinski CA, Lombardo F, Dominy BW, Feeney PJ. Experimental and computational approaches to estimate solubility and permeability in drug discovery and development settings. Adv Drug Deliv Rev 2001;46(1-3):3-26.

3. Lipinski CA. Drug-like properties and the causes of poor solubility and poor permeability. J Pharmacol Toxicol Methods 2000;44(1):235-49.

4. Attwood D, Florence AT. Surfactant systems: their chemistry, pharmacy, and biology. London, New York: Chapman and Hall; 1983.

5. Dangi JS, Vyas SP, Dixit VK. The Role of Mixed Micelles in Drug Delivery. I. Solubilization. Drug Dev Ind Pharm 1998;24(7):681-4.

6. Lehto P, Kortejärvi H, Liimatainen A, Ojala K, Kangas H, Hirvonen $\mathrm{J}$, et al. Use of conventional surfactant media as surrogates for FaSSIF in simulating in vivo dissolution of BCS class II drugs. Eur J Pharm Biopharm 2011;78(3):531-8.

7. Sun W, Larive CK, Southard MZ. A mechanistic study of danazol dissolution in ionic surfactant solutions. J Pharm Sci 2003;92(2):424-35.

8. Jamzad S, Fassihi R. Role of surfactant and $\mathrm{pH}$ on dissolution properties of fenofibrate and glipizide - A technical note. AAPS PharmSciTech 2006;7(2):E17-E22.

9. Gaucher G, Satturwar P, Jones MC, Furtos A, Leroux JC. Polymeric micelles for oral drug delivery. Eur $\mathrm{J}$ Pharm Biopharm 2010;76(2):147-58.

10. Henni-Silhadi W, Deyme M, Boissonnade M-M, Appel M, Le Cerf D, Picton L, et al. Enhancement of the solubility and efficacy of poorly water-soluble drugs by hydrophobicallymodified polysaccharide derivatives. Pharm Res 2007;24(12):2317-26.

11. Ménard N, Tsapis N, Poirier C, Arnauld T, Moine L, Gignoux $\mathrm{C}$, et al. Novel surfactants with diglutamic acid polar head group: Drug solubilization and toxicity studies. Pharm Res 2012;29(7):1882-96.

12. Torchilin VP. Micellar nanocarriers: Pharmaceutical perspectives. Pharm Res 2007;24(1):1-16.

13. Xiong XY, Li YP, Li ZL, Zhou CL, Tam KC, Liu ZY, et al. Vesicles from Pluronic/poly(lactic acid) block copolymers as new carriers for oral insulin delivery. J Control Release 2007;120(1-2):11-7. 
14. Sobczynski J, Smistad G, Hegge AB, Kristensen S. Molecular interactions and solubilization of structurally related mesoporphyrin photosensitizers by amphiphilic block copolymers (Pluronics). Drug Dev Ind Pharm 2015;41(8):1237-46.

15. Zhang N, Wardwell PR, Bader RA. Polysaccharide-Based Micelles for Drug Delivery. Pharmaceutics 2013;5(2):329-52.

16. Hostettmann K, Marston A. Saponins. New York: Cambridge University Press; 1995.

17. Guglu-Ustundag O, Mazza G. Saponins: Properties, applications and processing. Crit Rev Food Sci Nutr 2007;47:231-58.

18. Oakenfull DG. Aggregation of saponins and bile acids in aqueous solution. Aust J Chem 1986;39:1671.

19. Mitra S, Dungan SR. Micellar properties of Quillaja saponin. 1. Effects of temperature, salt and $\mathrm{pH}$ on solution properties. $\mathrm{J}$ Agric Food Chem 1997;45:1587-95.

20. Mitra S, Dungan SR. Micellar properties of Quillaja saponin. 2. Effect of solubilized cholesterol on solution properties. Colloids Surf B 2000;17:117.

21. Mitra S, Dungan SR. Cholesterol solubilization in aqueous micellar solutions of Quillaja saponin, bile salts, or nonionic surfactants. J Agric Food Chem 2001;49:384-94.

22. Wu YQ, Wang YL, Lei TZ, Xia YQ. The Solubilization Capability of Polycyclic Aromatic Hydrocarbons Enhanced by Biosurfactant Saponin Mixed With Conventional Chemical Surfactants. Petr Sci Tech 2014; 32(1):108-15.

23. Sasaki Y, Mizutani K, Kasai R, Tanaka O. Solubilizing properties of glycyrrhizin and its derivatives: solubilization of saikosaponin A, the saponin of Bupleuri Radix. Chem Pharm Bull 1988;36:3491-5.

24. Morita T, Nie RL, Fujino H, Ito K, Matsufuji N, Kasai R, et al. Saponins from Chinese curcubitaceous plants: solubilization of saikosaponin-a with hemslosides $\mathrm{Ma} 2$ and $\mathrm{Ma} 3$ and structure of hemsloside $\mathrm{H}$, from Hemsleya chinensis. Chem Pharm Bull 1986;34:401-5.

25. Watanabe K, Fujino H, Morita T, Kasai R, Tanaka O. Solubilization of saponins of Bupleuri Radix with ginseng saponins: cooperative effect of dammarane saponins. Planta Med 1988;54: 405-9.

26. Kimata H, Sumida N, Matsufuji N, Morita T, Ito K, Yata N, et al. Interaction of saponin of Bupleuri radix with ginseng saponin: solubilization of saikosaponin-a with chikusetsusaponin V (= ginsenoside- Ro). Chem Pharm Bull 1985;33:2849-53.

27. Zhou XH, Kasai R, Yoshikawa M, Kitagawa I, Tanaka O. Solubilization of saponins of Bupleuri Radix with ginseng saponins: effect of malonylginsenosides on aqueous solubility of saikosaponin-b1. Chem Pharm Bull 1991;39:1250-2.

28. Walthelm U, Dittrich K, Gelbrich G, Schöpke T. Effects of saponins on the aqueous solubility of different model compounds. Planta Med 2001;67(1):49-54.

29. Aungst BJ. Intestinal permeation enhancers. J Pharm Sci 2000;89(4):429-42.

30. Imai T, Sakai M, Ohtake H, Azuma H, Otagiri M. In vitro and in vivo evaluation of the enhancing activity of glycyrrhizin on the intestinal absorption of drugs. Pharm Res 1999;16:80-6.

31. Chao AC, Nguyen JV, Broughall M, Recchia J, Kensil CR, Daddona PE, et al. Enhancement of intestinal model compound transport by DS-1, a modified Quillaja saponin. J Pharm Sci 1998;87(11):1395-9.

32. Price KR, Johnson IT, Fenwick GR. The chemistry and biological significance of saponins in foods and feedingstuffs. Crit Rev Food Sci Nutr 1987;26(1):27-135.
33. Kawaguchi M, Kato T, Kamada S, Yahata A. Three-month oral repeated administration toxicity study of seed saponins of Thea sinensis L. (ryokucha saponin) in rats. Food Chem Toxicol 1994;32(5):431-42.

34. Qin Y, Wu X, Huang W, Gong G, Li D, He Y, et al. Acute toxicity and sub-chronic toxicity of steroidal saponins from Dioscorea zingiberensis C.H.Wright in rodents. J Ethnopharmacol 2009;126(3):543-50.

35. Oleszek W, Junkuszew M, Stochmal A. Determination and toxicity of saponins from Amaranthus cruentus seeds. J Agric Food Chem 1999;47(9):3685-87.

36. Malinow MR, McNulty WP, McLaughlin P, Stafford C, Burns $\mathrm{AK}$, Livingston $\mathrm{AL}$, et al. The toxicity of alfalfa saponins in rats. Food Cosmet Toxicol 1981;19(C):443-5.

37. Sun HX, Xie Y, Ye YP. Advances in saponin-based adjuvants. Vaccine 2009;27(12):1787-96.

38. Cox JC, Sjölander A, Barr IG. ISCOMs and other saponin based adjuvants. Adv Drug Deliv Rev 1998;32(3):247-71.

39. Kaukonen AM, Boyd BJ, Porter CJ, Charman WN. Drug Solubilization Behavior during in vitro Digestion of Simple Triglyceride Lipid Solution Formulations. Pharm Res 2004;21(2):245-53.

40. Stillhart C, Imanidis G, Kuentz M. Insights into drug precipitation kinetics during in vitro digestion of a lipid-based drug delivery system using in-line Raman spectroscopy and mathematical modeling. Pharm Res 2013;30(12):3114-30.

41. Pedersen BL, Mullertz A, Brondsted H, Kristensen HG. A comparison of the solubility of danazol in human and simulated gastrointestinal fluids. Pharm Res 2000;17(7):891-94.

42. Jiao J. Polyoxyethylated nonionic surfactants and their applications in topical ocular drug delivery. Adv Drug Deliv Rev 2008;60(15):1663-73.

43. Sarker DK. Engineering of nanoemulsions for drug delivery. Current Drug Deliv 2005;2(4):297-310.

44. Paul S, Kumar A, Yedurkar P, Sawant K. Design and development of multiple emulsion for enhancement of oral bioavailability of acyclovir. Drug Dev Ind Pharm 2013;39(11):1809-17.

45. Golemanov K, Tcholakova S, Denkov N, Pelan E, Stoyanov DS. Remarkably high surface visco-elasticity of adsorption layers of triterpenoid saponins. Soft Matter 2013;9(24):5738-52.

46. Bankefors J, Nord LI, Kenne LL. Structural classification of Quillaja saponins by electrospray ionization ion trap multiple-stage mass spectrometry in combination with multivariate analysis, proof of concept. Chemom Intell Lab Syst 2008;90:178-87.

47. Yan J, Wu Z, Zhao Y, Jiang C. Separation of tea saponin by two-stage foam fractionation. Sep Purif Technol 2011;80:300-5.

48. Sirtori CR. Aescin: Pharmacology, Pharmacokinetics, and Therapeutic Profile. Pharmacol Res 2001;44:183-93.

49. Asafu-Adjaye BE, Wong SK. Determination of Ginsenosides (Ginseng Saponins) in Dry Root Powder from Panax ginseng, Panax quinquefolius, and Selected Commercial Products by Liquid Chromatography: Interlaboratory Study. J AOAC Int 2003;86:1112-23.

50. Yamaguchi H, Matsuura H, Kasai R, Tanaka O, Satake M, Kohda H, et al. Analysis of Saponins of Wild Panax ginseng. Chem Pharm Bull 1988;36:4177-81.

51. Suhagia BN, Rathod IS, Sindhu S. Sapindus mukorossi (areetha): an overview. Int $J$ Pharm Sci Res 2011;2(8):1905-13. 
52. Petit PR, Sauvaire YD, Hillaire-Buys DM, Leconte OM, Baissac YG, Ponsin GR, et al. Steroid saponins from fenugreek seeds: Extraction, purification, and pharmacological investigation on feeding behavior and plasma cholesterol. Steroids 1995;60:674-80.

53. Yoshikawa $M$, Murakami $T$, Komatsu $H$, Murakami N, Yamahara J, Matsuda H. Medicinal foodstuffs. IV. Fenugreek seed. (1): structures of trigoneosides Ia, Ib, IIa, IIb, IIIa, and IIIb, new furostanol saponins from the seeds of Indian Trigonella foenum-graecum L. Chem Pharm Bull 1997;45:81-7.

54. Grover RK, Roy AD, Roy E, Joshi SK, Srivastava V, Arora SK. Complete 1H and 13C NMR assignments of six saponins from Sapindus trifoliatus. Magn Reson Chem 2005;43:1072-6.

55. Dinchev D, Janda B, Evstatieva L, Oleszek W, Aslani WR, Kostova I. Distribution of steroidal saponins in Tribulus terrestris from different geographical regions. Phytochemistry 2008;69:176-86.

56. Yan W, Ohtani K, Kasai R, Yamasaki K. Steroidal saponins from fruits of Tribulus terrestris. Phytochemistry 1996;42:1417-22.

57. Kiuchi F, Gafur A, Obata T, Tachibana A, Tsuda Y. Acacia concinna Saponins. II. Structures of monoterpenoid glycosides in the alkaline hydrolysate of the saponin fraction. Chem Pharml Bull 1997;45(5):807-12.

58. Food and Agriculture Organization. Quillaja extracts type 1 and 2: Chemical and Technical Assessment, 61st Report of the Joint FAO/WHO Expert Committee on Food Additives (JECFA), 2004.

59. Rangel-Yagui CO, Pessoa A Jr, Tavares LC. Micellar solubilization of drugs. J Pharm Pharm Sci. 2005;8(2):147-63.

60. Rosen MJ. Surfactants and Interfacial Phenomena. 3rd ed. New Jersey: John Wiley \& Sons, Inc. 2004.

61. Sanders MT, Brown LE, Deliyannis G, Pearse MJ. ISCOMTM_ based vaccines: The second decade. Immunol Cell Biol 2005;83(2):119-28. 